\title{
AC 2009-943: MATH TOOLS FOR ENGINEERING: A NEW APPROACH TO TEACHING CALCULUS III AND DIFFERENTIAL EQUATIONS
}

Hassan Moore, University of Alabama, Birmingham

Gregg Janowski, University of Alabama, Birmingham

Melinda Lalor, University of Alabama, Birmingham 


\title{
MATH TOOLS FOR ENGINEERING: A NEW APPROACH TO TEACHING CALCULUS III AND DIFFERENTIAL EQUATIONS
}

\begin{abstract}
During the fall of 2008 at the University of Alabama at Birmingham, a new course (EGR/MA 265 - Mathematical Tools for Engineering Problem Solving) was offered in a joint effort by the School of Engineering and the Department of Mathematics combining differential equations and calculus III into a four-hour semester-long course to aid in the transition of students from pure mathematics into engineering. This course was offered as an alternate track to the traditional calculus III and differential equations courses. Due to the time constraints within any one semester, a careful review of topics was made after interviewing every professor within the five departments of the School of Engineering who taught courses requiring calculus III or differential equations as a pre- or co-requisite. Initially, major areas to emerge were first-order linear ordinary differential equations; second-order linear ordinary differential equations with constant coefficients; multivariable functions, partial differentiation, and multiple integrals. The professors of the new course decided that they may be able to include integral theorems and an introduction to partial differential equations. In addition to the traditional material that these courses would typically provide, many application-based homework assignments and projects were given to provide engineering context. Each project assignment was given the same weight as a test but the projects were far more exploratory. Students were provided static notes in PDF format as well as dynamic notes in notebook player file format provided via the use of Mathematica Player by Wolfram to illustrate visually many of the concepts taught. Anonymous student comments via Individual Development and Educational Assessment (IDEA) surveys - an internal course evaluation tool - indicated that student perception of the course was generally positive.
\end{abstract}




\section{Introduction}

A new four semester-hour course has been offered at the University of Alabama at Birmingham which serves as an alternate to the traditional calculus III and differential equations courses. After receiving traditional calculus I and II courses which address the fundamentals of differentiation and integration, the new course, entitled "Mathematical Tools for Engineering Problem Solving," is intended to promote student engagement by emphasizing the use of mathematics in engineering applications rather than as a tool to be utilized later.

The intent of the new course is to implement more project- and inquiry-based study in order to foster deeper understanding of the fundamental theorems in multivariable calculus and differential equations. The new course (see Appendix A for content) was team taught by a Professor from the Mathematics Department, Dr. Gunter Stolz, and an Assistant Professor from within the School of Engineering, first author of this paper. Both instructors were involved in the initial planning of the course and all phases in between, along with members from the Mathematics Department and the School of Engineering. Thus, there was true collaboration in all aspects of the course. The need for calculus and differential equations reform within engineering has been noted for quite some time ${ }^{1,2}$.

In a recent article of the Chronicles of Higher Education ${ }^{3}$, it has been found that the engineering curriculum at many schools continue to remain far too theoretical. While this article points to the actual engineering courses within the curricula itself, the authors of this paper feel that the same is true with the manner in which students learn mathematics. Thus, there is a need for more integration that is inductive ${ }^{4}$ in approach. It is very difficult to simply flip a switch and have students solve engineering problems using mathematics, yet this is exactly what is attempted when students learn techniques in several courses and later are asked to act in an integrated sense. Bass ${ }^{5}$ states that "the phase transition...involves many partial shifts of focus from core mathematics toward applications and toward interdisciplinary work with the natural and social sciences, from academic to industrial and laboratory settings, from individual selfdirected work to collaborative and multidisciplinary effort, from technical communication with co-specialists to translational communication across disciplinary and cultural boundaries."

Many faculty members within the School of Engineering at the university were concerned with the severe lack of critical understanding of rudimentary concepts in calculus and differential equations. Students' basic mechanics were generally strong since students were very comfortable with equations once they took on the recognizable form shown in their earlier courses. However, there was a general lack of understanding of how to convert open-ended problems into solvable equations, use basic ideas in calculus such as recognizing a rate of change or accumulation, or how new areas in science make use of calculus.

Due to the reduction in credits hours (from seven to four) and a desire to achieve greater depth of understanding within the areas identified by the engineering faculty, the topical breadth of the 
course was limited, much like an optimization problem in which an ideal amount of breadth and depth is sought. If every area within the original two courses was deemed critical, there would be far too much material to attain any level of understanding.

Also given in this paper are some of the student responses from the Individual Development and Educational Assessment (IDEA) surveys, which the university uses for instructor/course evaluation. While this may be viewed by some as inherently subjective information, the authors feel that there is value in illustrating the perception of the students who had initially taken the course. A recognized weakness of the survey results may be that students cannot compare the course with the alternative path in the Mathematics Department since they chose one and not the other. However, students have taken mathematics courses in the past and have talked to friends in the other traditional courses such that they would understand the key advantages and disadvantages to the new course and know whether or not it was effective. Plus, future feedback from faculty serving both students from the new course and the traditional track will clarify any discrepancies in which more discussion would ensue as to how to solve the problem.

While the professors who taught the course were pleased with the first iteration of the course, there was still room for growth and change identified. For instance, the professors thought that too much time was spent on quizzes when a more prudent use of the time could have been to actively engage students periodically using pause drills in which students attempt problems with guidance from their peers and professors. The active engagement gives students an early attempt to gain confidence in their understanding. Another improvement might be to focus more on vector differential calculus and a little less on multiple integrals.

\section{Results and Discussion}

There were many challenges faced in the initial offering of the course. First and foremost, the material to be taught turned out to be overly ambitious. Although engineering faculty from each of the five major disciplines (Mechanical, Biomedical, Electrical, Materials, and Civil) who listed calculus III and differential equations as a pre- or co-requisite would choose the primary topic areas, the professors teaching the course initially thought that they would have time to cover at least one integral theorem (Green's Theorem) - if not all three (Green's, Stokes' and Gauss' Theorems) - and give an introduction to partial differential equations along with the separation of variables technique. These were not items specifically mentioned in the initial interviews but are considered important mathematical topics.

It soon became apparent that there would not be enough time to teach any of the integral theorems or partial differential equations though it was initially reported ${ }^{6}$ that the focus would be on first-order ordinary differential equations, second-order ordinary differential equations, multivariable differentiation and integration, integral theorems, and an introduction to partial differential equations. The integral theorems and partial differential equations would not be attempted if it meant that depth were to be sacrificed. 
Another critical component in the design of the course was the textbook. Criteria for the textbook include (1) logical flow with the design of the course, (2) easy for advanced freshmen and sophomore students to read and understand, (3) useful as a reference text for later use, (4) contains the differential equations and calculus topics identified as critical, and (5) includes clear examples for students to follow. In an ideal world, the textbook would also have an associated homework delivery system to provide the practice medium and immediate feedback necessary for basic competence and concrete understanding on which to build abstract thought.

Thus, the professors gave serious consideration to the following textbooks:

Kreyszig, Erwin. Advanced Engineering Mathematics. $9^{\text {th }}$ ed. New York: John Wiley \& Sons, 2006.

O O’Neil, Peter. Advanced Engineering Mathematics. $6^{\text {th }}$ ed. Boston: Thompson, 2007.

- Zill, Dennis G. and Cullen, Michael R. Advanced Engineering Mathematics. $3^{\text {rd }}$ ed. Sudbury: Jones and Bartlett, 2006.

○ Harman, Thomas L., Dabney, James, and Richert, Norman. Advanced Engineering Mathematics with MATLAB. $2^{\text {nd }}$ ed. Boston: Thompson, 2000.

As a potential supplement,

○ Hunt, Brian R., Lipsman, Ronald L., Osborn, John E., and Rosenberg, Jonathan M. Differential Equations with MATLAB. $2^{\text {nd }}$ ed. New York: John Wiley \& Sons, 2005.

The Zill/Cullen textbook would ultimately be chosen as the best fit for our students, especially since the Mathematics Department already used Dennis Zill's "A First Course in Differential Equations" textbook for the Differential Equations course. None of the textbooks offered a homework delivery system. It was clear that there are not many textbooks that exactly cater to a course like this, let alone provide a homework delivery system. So, the professors decided to use Blackboard Vista, a course management and homework delivery system internally supported by the university in which the professors were free to create their own homework problems. The textbook was primarily used to give extra problems to students though they were never turned in for a grade.

While Blackboard was not ideal, it offered students a clear advantage to turning in an assignment and waiting for the results. Frequently, the class had moved beyond the homework material by the time students would receive feedback on homework assignments graded by the professor, leaving students clueless as to their performance in a certain area. Also, using Blackboard as the homework delivery system alleviated the time-consuming task of grading assignments, which often leads to homework not being collected. With the team-teaching aspect, students were allowed ample opportunities to see either professor with any problems from their homework. There were also active-learning ${ }^{7,8}$ possibilities beyond the projects. There were several pause drills in which students were allowed to get experience with the material while breaking up long 
periods of lecture when students may begin to tune out ${ }^{9}$. Although voting paddles or clickers were not ready for the initial course offering, it appears that they are forthcoming and will be immediately integrated into the course. This addition will allow students to participate in class without fear of ridicule for erroneous responses and allow professors a real-time sense whether there is student comprehension.

Another tool used was guided note-taking ${ }^{8}$, in which the professors provided PDF notes and Mathematica Player notes which would include blank areas for examples to be solved or missing words or statements for key terminology. All examples were left for either the professor to cover or for the student to perform as an active pause drill.

During one of the class sessions, one of the professors built a small RC-circuit with various switchable resistors to illustrate the ability of the students to use Kirchhoff's second law, develop the differential equation, and find the time constant based on the source, capacitance, and resistance values. Students predicted the potential across the capacitor for the time constant found, confirming the accuracy of their calculation using their newly acquired skills in solving second-order differential equations with constant coefficients.

Another exercise that the professors believe has merit and will continue throughout future course offerings was having the students draw their own 11x11 direction field. An 11 x 11 grid of equally spaced points was provided onto which students would draw small lineal elements (slopes of a function) to illustrate the infinite solutions possible. Using an autonomous field in which the slope function depends solely on one variable really drove home autonomous differential equations since the students learned quickly to avoid calculating 121 slopes. They also learned the meaning of a unique solution passing through a given point in the field, initial value problems, and the power of a computer algebra system to serve as an aid in visualizing solutions to differential equations.

The justification, expected learning outcomes, pedagogy and instruction, and assessment of the course prior to the first full implementation may be found in the earlier work by the authors ${ }^{6}$. While the information remains accurate, there were several things learned after the first iteration of the course which some may find debatable. The learning outcomes from the initial paper will be re-stated here.

Students who complete the newly designed course will be able to:

1. Evaluate engineering problems and choose the tools (from basic sciences and mathematics) required for solving.

2. Utilize appropriate units, constraints, physical constants and associated equations in the solution of engineering problems.

3. Demonstrate an intuition about engineering problems including relationships among variables and mathematical connections. 
4. Assess the validity of the mathematical solution using units, physical intuition, and boundary conditions.

5. Categorize engineering problems by common solution structures

6. Utilize a Computer Algebra System (CAS), such as Mathematica, to solve engineering problems.

7. Demonstrate mathematical concepts and computational skills in differential equations and two-and three-dimensional calculus

8. Solve fundamental mathematical problems in each area addressed by the course.

9. Develop the numerical confidence necessary to tackle significant engineering problems and defend their approach and solution.

Again, the nine items were pretty accurate for the course. There may be a slight change in thinking about points one and nine, if it can be considered much of a change at all. Though the thinking regards subtle lines of change, a little discussion may be warranted.

In the first point, it is mentioned that students would choose the mathematics tool necessary. It is not the intent to state that there will be a plethora of choices in which students will have to make the right choice in solving a problem. As in a traditional mathematics course, students were typically offered homework problem sets immediately following instruction of a key area though problems from earlier areas would thread their way throughout the assignments given in the course. The projects also followed the key ideas in which there was little guesswork regarding the topic area even though students were asked to go beyond what is typical in a textbook problem, often confronting areas in which they may have been weak in the past - such as McLaurin or Taylor expansions and integrating rational functions containing partial fractions or trigonometric substitutions - or had little experience at all - such as solving transcendental equations (most students did not recall Newton's Method from their first calculus course).

In point nine, it may be argued that the numerical confidence may be the same as the students taking the traditional track. It is very difficult within the time constraints of a semester to do much numerically. Most differential equations courses may touch upon Euler's method but a complete numerical analysis course would be needed to provide numerical confidence to tackle significant engineering problems. So, it may be argued that it is near impossible, even in the traditional differential equations course, to develop a keen sense of numerical intuition without a full blown numerical methods course, much like the lesson learned when a transcendental was placed into a project assignment and many students did not know how to solve it.

There were 50 students taking the course, each remaining with the course throughout the semester. There were 42 students who would go through the traditional track, taking calculus III during the same semester. It should be mentioned that students chose their own track; they were 
not advised to take one course or the other based on the ability of the student or some other criteria.

The grade distribution at the end of the course was 15 A's, 19 B's, 11 C's, 3 D's, and 2 F's. Again, it should be mentioned that the senior instructor of the course is a fully tenured professor from the Mathematics Department with no appointment within the School of Engineering. He was fully committed to ensuring that students understood the material.

\section{Project-Based Learning ${ }^{10}$}

There were four projects given to the students during the semester (publication is forthcoming). Students were given roughly two to three weeks to complete each project which focused on one of the major areas of the course. The professors feel that they are self-contained, easy to integrate into a course covering similar general topics, and are very instructive. Also, to avoid having future students collect a repository of old projects waiting to copy the results, new projects are being generated each semester. The number of projects has now been cut to three per semester due to the fact that they are extremely time-consuming to create as well as grade. Even with team-teaching, Dr. Stolz would comment that this course required more of his time than any course prior.

The titles of the four projects given during the first iteration were

(1) Free fall, air resistance, and parachutes (First-Order Differential Equations),

(2) A model of resonance for car suspensions (Second-Order Differential Equations),

(3) Minimizing heat loss (Partial Derivatives), and

(4) Moments of inertia (Multiple Integrals).

The projects were quite comprehensive and provided great insight into student understanding of the major topic areas. While student collaboration was encouraged, the professors teaching the course demanded individual reports. Another important criterion was to have the projects such that they may be implemented into any course at any university teaching these topics.

Students expressed a lot of satisfaction with the projects, especially the components which made them take a physical role in the project. For instance, in the project on car suspensions students needed to provide the year, make and model of their vehicle (or a friend's vehicle) to find an "effective spring constant" for the lumped mass of the vehicle by sitting somewhere near the center of the vehicle and recording the displacement. This information was later used to calculate the speed at which students would achieve resonance on certain roads. Ultimately, students reported that they were having so much fun in this project that they decided to add a second person on top of the vehicle because they knew that Hooke's Law was linear and they wanted to see if they would get the same spring constant. This extension was a clear indication of the natural learning tendencies that many of the students share when learning is both active and relevant. 
There were negative points that should be mentioned. First, this course may not be used in pursuit of a math minor, if a student were so inclined even though the Mathematics Department did approve the course to serve as a pre-requisite for three courses - vector analysis, complex analysis, and transforms. Second, students typically performed poorer in the application-based problems on homework and tests compared to the traditional theoretical problems. It was realized that many students have adapted to the clinical method in which mathematics is taught and found some difficulty in problems that require some physics or chemistry. Also, the difficulty in grading the projects should not be understated. It was extremely arduous but ultimately seen as well-worth the results as the professors are confident that they are sending along improved students who definitely learned the topics introduced, especially to the level at which their grades reflect.

Many offline comments were made at the end of the course by students who believed that the amount of time needed to solve the projects added an extra dimension to the course that would increase the difficulty of the semester, especially since they typically carried a full load. But they also mentioned that they truly learned a lot in the course as seen in some of the student comments taken from the IDEA survey listed below.

It should be mentioned that there were several simple "yes" answers that were left out of each category. There were also blank responses since comments are at the discretion of the student and not every student will comment on a course.

\section{Student Responses to the course}

\section{Was the balance of lecture and other in-class teaching methods right in the course? (Explain).}

Yes, we were able to get familiar with both the math theory and the applications of the certain theories.

I believe the balance of lecture and other methods of teaching were correct.

There was more mathematics than applications, but seemed necessary to grasp concepts.

A little rocky at first, but the course was experimental. Once they figured out how to go about teaching the class, it was very effective. If something wasn't clear in class for whatever reason, going to either of the instructors' offices took care of that.

It seemed like a heavy load for both professors, and I would see why. But these guys did one Somalia of a job.

Yes, it seemed as though Dr. Moore was the applications man and Stolz was the mathematician. However, both were capable of the other's job and did an excellent job teaching. 
Yes, while one taught the theories behind the concept, the other applied the material in a real-world situation. The two teaching methods of both instructors were coupled very well to each other.

Yes, it was a good balance. They were able to give us problems to work with class members after explaining them, and that helped. Both instructors had their own way of teaching, and each one was efficient to me.

Yes. Everything we did in class was geared towards us understanding the subject better.

I thought there was a good balance and the material in the projects was covered in depth and clearly understood before attempting the challenging projects. Dr. Stolz was very clear at explaining the mathematical theory and Dr. Moore was well versed and able to explain the applications. I also think using Mathematica was very helpful by showing us visualizations of concepts hard to grasp without actually seeing it and also by showing us how important technology is to our field of study

Yes, it made it easier to pay attention without it getting dull

Yes. The use of Mathematica is great for us to visualize things.

\section{Was the balance of theory and applications right for the course (both instructors)? Explain.}

Yes, the projects helped with theory and applications learned from the notes and book.

Yes, projects were given to allow for testing us on applications and tests were given to test theory.

Theory and applications were not overdone although some of the applications were difficult to get through.

Yes. The balance was right. I felt like the application were the part we really needed to understand. But the theory had to be taught just enough for us to understand what we were doing. The application mind set had to be used more for this course instead of just working a bunch of problems by a memorized pattern, which was nice.

Yes. It was cool because we saw the mathematical side and the engineering side of the math. It worked well. The teachers worked well together and did their best to help us understand what was going on.

Yes, the balance between math lecture and Dr. Moore's applications worked well. I was able to understand the math concepts better.

Yes, a good mix that helped tie theory to reality. 
I'd say so. Almost all the theory was eventually used in an application in a project. I'd say that's balanced.

Stolz seemed to do more theory and Moore did more applications. It all seemed to work well for the class. We were tested fairly on what was covered in class. The projects are a little much, with the homework, tests, and 4 other classes to prepare for.

Yes, I thought it worked well going back and forth between learning the math and the applications. Knowing how the math is applied gave me more incentive to learn it, and made it more interesting when I know how it could be used.

Yes, being an engineering course it is important to study applications, but to do those you have to have a base in theory

Yes, it helped me to know more about what kind of questions Engineers solve and how to solve them myself.

Yes-the balance was fine. Dr. Stolz explained everything well and Dr. Moore supported the claims suggested by Dr. Stolz with realistic applications.

Yes, but in the beginning it was a little confusing. I just had to get use to it.

Yes, we did enough example practice and gained enough basic principle and definitions before we moved on to the application.

\section{Comments: Use the space provided in the text area below for your comments.}

Enjoyed this class

Thanks for the class!

I enjoyed the course and would take another course from either and/or both teachers again.

Great course.

All in all, I enjoyed the class and feel that I understand most the material.

I enjoyed this class. The teachers are both great. They are willing to meet, give help, and are reasonable with deadlines for assignments. I have nothing but positive comments for both teachers and this course.

Unfortunately, this class, because of the amount of out of out of class work involved (projects and homework), took away a little too much time from my other classes.

This course was an excellent course and I will recommend it to other students 
Overall good class but there were several times that I would have a Statics test or other demanding test right when a project would be due that made it especially difficult. I feel as though I and my friend's grades were almost jeopardized due to this. This NEEDS to be drastically taken into consideration when evaluating next course (I mean, 4 projects, 4 test, 6 quizzes, and 10-12 homework assignments is a bit much, come on...) Other than that, perfectly satisfactory.

\section{Summary}

A new course has been developed at the University of Alabama at Birmingham in which students are given rigorous training in the application of mathematical ideas. Several components of the course have been outlined. During the fourteen weeks in the semester, everything that was outlined by the faculty within the School of Engineering was covered. The material initially added by the professors of the course, namely the integral theorems and the introduction to partial differential equations, was not completed due to time constraints. They remain lofty goals at best. Nearing the end of the semester, it was decided that the need to spend more time on the previous areas and remaining project was more beneficial. In essence, the extent of the material covered would include solving first-order ordinary differential equations using the separable and linear methods; solving second-order ordinary differential equations with constant coefficients using undetermined coefficients and variation of parameters; and partial derivatives, line integrals, double integrals, triple integrals, cylindrical and spherical coordinates. For a full listing of topic areas taught, see Appendix A.

Some of the areas omitted from the typical calculus III and differential equations track were Laplace transforms, the Jacobian, surface integrals, and the integral transforms (Green's Theorem, Stokes' Theorem, and Gauss' Theorem). Of the above areas, Laplace transforms were mentioned as an area of heavy need in the preliminary interviews but it was decided that the departments would handle the topic with the extra three hours given to the department. Besides, Laplace transforms were being taught or re-taught in the departments already. For instance, in electrical engineering, there is a formal course which handles the Laplace, Fourier, and Ztransform methods. Regarding the other areas, there was a general lack of use by any of the departments as no one suggested their inclusion. 


\section{Bibliography}

1. Ruane, M., "Calculus Reform, Differential Equations and Engineering", Proceedings of the Annual Conference and Exposition, American Society for Engineering Education, 2001.

2. Mumford, D., Calculus Reform - for the Millions, Notices of the AMS, Vol. 44, No. 5, May 1997.

3. Basken, P., Why Engineering Schools are Slow to Change, Chronicles of Higher Education, January 2009.

4. Prince, M. J. and Felder, R. M., Inductive Teaching and Learning Methods: Definitions, Comparisons, and Research Bases, Journal of Engineering Education, April 2006.

5. Bass, H., Mathematicians as Educators, American Mathematics Society, Vol. 44, No. 1, January 1997.

6. Janowski, G., Lalor, M., and Moore, H., "New Look at the Upper-Level Mathematics Needs in Engineering Courses at the University of Alabama at Birmingham", Proceedings of the $115^{\text {th }}$ Annual Conference and Exposition, American Society for Engineering Education, Pittsburgh, PA, June 22-25, 2008.

7. Prince, M. J., Does Active Learning Work? A Review of the Research, Journal of Engineering Education, July 2004.

8. Silberman, M., Active Learning: 101 Strategies to Teach Any Subject, Allyn \& Bacon, 1996.

9. Polio, H.R., What Students Think About and Do in College Lecture Classes. Teaching-Learning Issues No. 53. Knoxville: Learning Research Center, University of Tennessee, 1984.

10. Srinivasan, M., Wilkes, M., Stevenson, F., Nguyen, T., and Slavin, S., Comparing Problem-Based Learning with Case-Based Learning: Effects of a Major Curricular Shift at Two Institutions, Academic Medicine, Vol. 82, No. 1, January 2007. 
Appendix A: Full list of topics actually covered in EGR/MA 265.

\section{Part I: First-Order Ordinary Differential Equations}

Chapter 1: Introduction to Ordinary Differential Equations

- Definition of a Differential Equation

- Models of Real Systems or Phenomena

- Order of a Differential Equation

- Linear Ordinary Differential Equations

- Solutions of a Differential Equation

- Direction Fields

- Initial Value Problems

- Existence and Uniqueness of Solutions for Initial Value Problems

Chapter 2: Solution Methods for First-Order Ordinary Differential Equations

- Definition (Separable Equation)

- Solution of a Separable Equation of Type I

- Definition (Linear Equation)

- Solving the Homogeneous Linear Equation

- Solving the Inhomogeneous Linear Equation

Chapter 3: Applications of First-Order Ordinary Differential Equations

- Growth and Decay

- Newton's Law of Cooling

- Series Circuits

- The Logistic Equation

\section{Part II: Second-Order Linear Des and Their Applications}

Chapter 4: General Theory of Higher Order Linear Differential Equations

- Initial-Value Problems

- Existence and Uniqueness

- Homogeneous Equations

- Superposition Principle

- Linear Combinations

- Linear Dependence/Independence

- Nonhomogeneous Equations 
Chapter 5: Second-Order Linear Ordinary Differential Equations with Constant Coefficients

- The Auxiliary Equation

- Case I: Distinct Real Roots

- Case II: Repeated Real Roots

- Case III: Complex Conjugate Roots

- Important Special Cases

- The Method of Undetermined Coefficients

- Variation of Parameters

Chapter 6: Applications of Second-Order Linear Ordinary Differential Equations

- Spring/Mass Systems

- Free Undamped Motion

- General Solution to Free Undamped Motion

- An Alternative Form of Harmonic Motion

- Free Damped Motion

- Overdamped Systems

- Critically Damped Systems

- Underdamped Systems

- Driven Motion

- Transient and Steady-State Solutions

- Driven Motion without Damping

- Pure Resonance

- Series Circuit Analogue LRC Example

\section{Part III: Multivariable Functions: Their Derivatives and Integrals}

Chapter 7: Partial and Directional Derivatives

- Functions of Two and Three Variables

- Level Curves

- Partial Derivatives and Higher-Order Partial Derivatives

- Equality of Mixed Partial Derivatives

- Chain Rule and Gradient

- Computing the Directional Derivative

- Direction of Steepest Ascent

- Level Surfaces in $\mathbb{R}^{3}$

- Tangent Planes to Level Surfaces 
Chapter 8: Line Integrals

- Parametric Curves in $\mathbb{R}^{2}$

- Line Integrals in $\mathbb{R}^{2}$

- Geometric Meaning of $\int_{C} G(x, y) d s \&$ Work

- Line Integrals over Closed Curves

- Line Integrals in $\mathbb{R}^{3}$

- Conservative Force Fields in $\mathbb{R}^{2}$

- Work in Conservative Force Fields

- A Criterion for the Existence of a Potential

- Conservative Forces in $\mathbb{R}^{3}$

- The Coulomb Potential

Chapter 9: Double and Triple Integrals

- Definition of a Double Integral

- Evaluating Double Integrals

- Center of Mass of a Lamina

- Moments of Inertia of a Lamina

- Triple Integrals

- Applications of Triple Integrals

- Double Integrals in Polar Coordinates

- Cylindrical and Spherical Coordinates 